activity in adipose tissue. The studies cannot resolve whether human PPARG mutations in FPLD3 might act in a dominant negative manner to interfere with function of the normal allele product or whether haploinsufficiency of PPAR $\gamma$ activity is more important. Interestingly, simple haploinsufficiency of PPAR $\gamma$ activity in mice by removal of one Pparg allele actually protects against insulin resistance (15), supporting the idea that missense mutations have distinct effects compared with simple reduction in PPAR $\gamma$. In any event, the findings of Tsai et al. reinforce the importance of PPAR $\gamma$ in adipogenesis (4), highlight the role of adipose tissue as an endocrine organ (16), and also support the idea that PPARG mutations affect metabolic and vascular phenotypes through multiple mechanisms, some of which are distinct from effects on adipose tissue mass or distribution.

Address correspondence to: Robert A. Hegele, Blackburn Cardiovascular Genetics Laboratory, Robarts Research Institute, 406-100 Perth Drive, London,
Ontario N6A 5K8, Canada. Phone: (519) 663-3461: Fax: (519) 663-3037; E-mail: hegele@robarts.ca.

1. Laaksonen, D.E., et al. 2002. Metabolic syndrome and development of diabetes mellitus: application and validation of recently suggested definitions of the metabolic syndrome in a prospective cohort study. Am. J. Epidemiol. 156:1070-1077.

2. Lakka, H.M., et al. 2002. The metabolic syndrome and total and cardiovascular disease mortality in middle-aged men. JAMA. 288:2709-2716.

3. Expert Panel on Detection, Evaluation, and Treatment of High Blood Cholesterol in Adults. 2001. Executive summary of the third report of the National Cholesterol Education Program (NCEP) expert panel on detection, evaluation, and treatment of high blood cholesterol in adults (Adult Treatment Panel III). JAMA. 285:2486-2497.

4. Marx, N., Duez, H., Fruchart, J.C., and Staels, B. 2004. Peroxisome proliferator-activated receptors and atherogenesis: regulators of gene expression in vascular cells. Circ. Res. 94:1168-1178.

5. Stumvoll, M., and Haring, H.U. 2002. Glitazones: clinical effects and molecular mechanisms. Ann. Med. 34:217-224.

6. Hegele, R.A. 2003. Monogenic forms of insulin resistance: apertures that expose the common metabolic syndrome. Trends Endocrinol. Metab. 14:371-377.

7. Tsai, Y.-S., et al. 2004. Hypertension and abnormal fat distribution but not insulin resistance in mice with P465L PPAR $\gamma . J$. Clin. Invest. 114:240-249. doi:10.1172/JCI200420964.

8. Barroso, I., et al. 1999. Dominant negative muta- tions in human PPARgamma associated with severe insulin resistance, diabetes mellitus and hypertension. Nature. 402:880-883.

9. Agarwal, A.K., and Garg, A. 2002. A novel heterozygous mutation in peroxisome proliferatoractivated receptor-gamma gene in a patient with familial partial lipodystrophy. J. Clin. Endocrinol. Metab. 87:408-411.

10. Hegele, R.A., et al. 2002. PPARG F388L, a transactivation-deficient mutant, in familial partial lipodystrophy. Diabetes. 51:3586-3590.

11. Savage, D.B., et al. 2003. Human metabolic syndrome resulting from dominant-negative mutations in the nuclear receptor peroxisome proliferator-activated receptor-gamma. Diabetes. 52:910-917.

12. Hegele, R.A., et al. 2003. Elevated serum C-reactive protein and free fatty acids among nondiabetic carriers of missense mutations in the gene encoding lamin $\mathrm{A} / \mathrm{C}(L M N A)$ with partial lipodystrophy. Arterioscler. Thromb. Vasc. Biol. 23:111-116.

13. Hegele, R.A. 2004. Phenomics, lipodystrophy and the metabolic syndrome. Trends Cardiovasc. Med. 14:133-137.

14. Dobrian, A.D., Schriver, S.D., Khraibi, A.A., and Prewitt, R.L. 2004. Pioglitazone prevents hypertension and reduces oxidative stress in diet-induced obesity. Hypertension. 43:48-56.

15. Miles, P.D., Barak, Y., He, W., Evans, R.M., and Olefsky, J.M. 2000. Improved insulin-sensitivity in mice heterozygous for PPAR-gamma deficiency. J. Clin. Invest. 105:287-292.

16. Engeli, S., et al. 2003. The adipose-tissue reninangiotensin-aldosterone system: role in the metabolic syndrome? Int. J. Biochem. Cell Biol. 35:807-825.

\title{
Innate immunity dictates cytokine polarization relevant to the development of pulmonary fibrosis
}

\author{
Robert M. Strieter and Michael P. Keane
}

\author{
Departments of Medicine, Pathology, and Pediatrics, Division of Pulmonary and Critical Care Medicine, \\ The David Geffen School of Medicine at the University of California, Los Angeles, Los Angeles, California, USA.
}

\begin{abstract}
New data support the importance of the innate immune response in the resolution or progression of pulmonary fibrosis. The presence of CXC chemokine receptor 3-expressing cells, specifically pulmonary NK cells, is necessary to produce IFN- $\gamma$. This is critical in the polarization of the immune response to injury toward a favorable Th1 response and resolution. In contrast, a Th2 response is associated with progressive fibrosis (see the related article beginning on page 291).
\end{abstract}

Pulmonary fibrosis is a host response to a variety of known and idiopathic processes and is clinically characterized by insidious onset of dyspnea and abnormal lung func-

Nonstandard abbreviations used: bronchoalveolar lavage (BAL); CXC chemokine receptor 3 (CXCR3); idiopathic pulmonary fibrosis (IPF); usual interstitial pneumonia (UIP).

Conflict of interest: The authors have declared that no conflict of interest exists.

Citation for this article:

J. Clin. Invest. 114:165-168 (2004).

doi:10.1172/JCI200422398 tion. The pathology of pulmonary fibrosis demonstrates features of dysregulated and abnormal repair with exaggerated vascular remodeling, fibroproliferation, and deposition of extracellular matrix, all leading to progressive fibrosis and loss of lung function. However, the most devastating form is idiopathic pulmonary fibrosis (IPF). IPF is a chronic and often fatal pulmonary disorder with a mortality rate as high as $70 \%$ five years after diagnosis $(1,2)$. The prevalence of IPF increases with age (2, $3)$. There has been an increase in the inci- dence of this disorder over the last 10-15 years in many Western countries, including the United Kingdom, New Zealand, and Germany (3). The inability of current immunosuppressive therapies to alter the prognosis of this disorder has renewed scientific interest in multiple areas and has raised additional questions as follows: (a) why are certain individuals susceptible to this disease? do genetic, environmental, or a combination of these factors contribute to the pathogenesis of this disorder? (b) why is the host response to injury to the alveolar capillary wall in susceptible individuals predominated by fibrosis and loss of alveolar architecture instead of resolution? and (c) what are the initial events within the host in response to injury that ultimately set the stage for polarization of the response toward either resolution or perpetuation of fibrosis? 


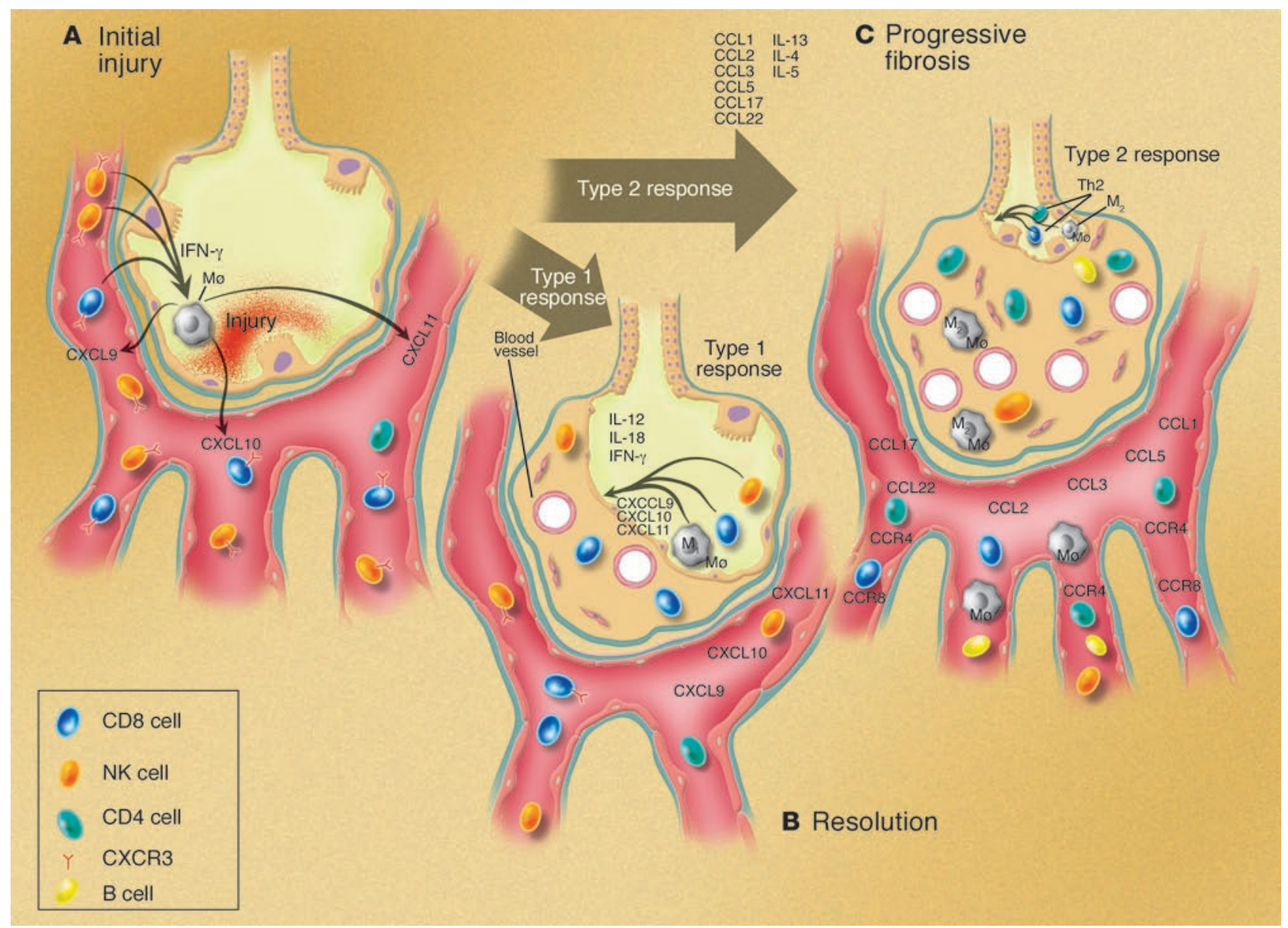

Figure 1

Polarization of the inflammatory response in pulmonary fibrosis. (A) Polarization of the immune response to injury is critical in dictating the ultimate response to that injury. (B) A type 1 response (i.e., cell-mediated immunity) leads to resolution. (C) In contrast, a type 2 response (i.e., humoral-mediated immunity) is associated with progressive fibrosis. CCL1, CC chemokine ligand 1; CXCL9, CXC chemokine ligand 9.

\section{Innate immunity and control of pulmonary fibrosis}

To begin to address the latter question, in this issue of the JCI, Jiang and associates report that $\mathrm{CXC}$ chemokine receptor 3 (CXCR3) is important in the regulation of pulmonary fibrosis (4). The results of this study shed new light on the importance of the initial innate host-immune response to injury, which ultimately sets the stage for polarizing the injurious response toward either resolution or progressive pulmonary fibrosis. By using CXCR3-deficient mice as compared to wild-type mice exposed to the pulmonary fibrosing agent bleomycin they were able to demonstrate that CXCR3-deficient mice exhibited progressive interstitial fibrosis without an increase in inflammatory cell recruitment. In fact, they found a selective defect in the recruitment of $\mathrm{CD}^{+}$ lymphocytes and NK cells into the lungs of CXCR3-deficient mice in response to bleomycin. Moreover, they found a previously unrecognized, profound defect in the number of pulmonary NK cells at baseline in CXCR3-deficient mice.

The defect in the presence of $\mathrm{CD}^{+} \mathrm{T}$ cells and NK cells in response to bleomycininduced lung injury in CXCR3-deficient mice was associated with a marked reduction in both the early (within 24 hours) burst of IFN- $\gamma$ and the subsequent expression of the CXCR3 ligand CXCL10 in the bronchoalveolar lavage (BAL) (4). To specifically demonstrate that the reduction in the early expression of IFN- $\gamma$ played a pivotal role in mediating progressive interstitial fibrosis in CXCR3-deficient mice, the investigators performed three elegant studies. First, they demonstrated that reconstitution of CXCR3-deficient mice with exogenous IFN- $\gamma$ for the first 48 hours after bleomycin exposure resulted in markedly attenuated pulmonary fibrosis. Second, they demonstrated that administration of neutralizing anti-IFN- $\gamma$ antibody prior to exposure to bleomycin significantly increased pulmonary fibrosis in wild-type mice. Finally, they used adoptive transfer of CXCR3-expressing mononuclear cells from wild-type mice to restore endogenous IFN- $\gamma$ in CXCR3-deficient mice and found reduced pulmonary fibrosis in response to bleomycin. Taken together, these data support the notion that the presence of CXCR3-expressing cells, specifically pulmonary NK cells, is both necessary and sufficient to produce IFN- $\gamma$, which is ultimately critical in polarizing the immune response to injury towards resolution rather than progressive fibrosis. 


\section{Clinical implications and future directions}

How do the findings for CXCR3-deficient mice and the importance of IFN- $\gamma$ translate to the study of human pulmonary fibrosis and the pathogenesis of IPF? The histopathology of IPF is classified as usual interstitial pneumonia (UIP) (5-7). UIP consists of a temporal heterogeneity of lung tissue; areas of normal lung tissue are present in addition to fibroblastic foci resulting from recent active fibrosis and honeycomb cysts resulting from older fibrotic lesions. This description suggests that areas of the lung have been subjected to injury at different times, resulting in both new organizing fibrosis and established fibrotic areas of the lung within the same low-power microscopic view. This histopathological pattern suggests that the host is responding to multiple hits, resulting in a predominance of fibrosis and loss of lung architecture. However, neither the initial injurious event nor the mechanisms responsible for the perpetuation of fibrosis are known. If the multiple hits over time in IPF are related to persistence or reexposure to specific antigens (i.e., environmental toxins, microbes, or self antigens) in the lungs of susceptible individuals, then failure to mount an appropriate type 1 cytokine response (i.e., IFN- $\gamma$; Th1) will lead to inability to eradicate the antigen. This situation will shift the polarization of the innate-adaptive immune response from type 1 (i.e., cellmediated immunity) toward type 2 (i.e., humoral-mediated immunity) with the added component of exuberant fibrosis in an attempt to contain the antigen.

Is there any evidence to support the contention that there is a type 2 immune response to specific antigens in patients with IPF? Recent microarray analysis of genes in IPF lung tissue has demonstrated that specific genes that are clustered in the following functional categories are substantially upregulated: smooth muscle markers; extracellular matrix proteins, growth factors, and proteases; cytokines, chemokines, and antioxidants; complement, amyloid, and immunoglobulins (8). The expression of genes encoding immunoglobulins and extracellular matrix proteins supports the potential contention that there is a host response to an antigen or antigens with polarization toward a type 2 response. In further support of this notion is the recent multicenter study that demonstrated marked mediastinal lymphadenopathy in patients with a pathological diagnosis of IPF (9). Taken together, despite the current belief that IPF is not an immunologically driven disorder, the above data support the contention that there is a predominant type 2, antigen-driven immune response with upregulated profibrotic genes, expression of immunoglobulin genes, and evidence of lymphoproliferation in response to a potential unknown antigen.

What are the potential mechanisms by which a CXCR3/CXCR3-dependentIFN- $\gamma$ biological axis may be beneficial in patients with IPF? CXCR3 is expressed on Th1 and NK cells (10-13). CXCR3 ligands, such as CXCL9, CXCL10, and CXCL11, are critical for trafficking of CXCR3expressing cells under conditions of type 1 cytokine-mediated immune responses (10-13). Therefore, CXCR3/CXCR3 ligands will predominantly recruit $\mathrm{Th} 1$ cells to the local microenvironment and lead to further amplification of the expression of endogenous IFN- $\gamma$. IFN- $\gamma$ is the most pivotal type 1 cytokine and it has the known ability to promote both type 1 , cell-mediated immunity and concomitant attenuation of fibrosis (14). A recent multicenter study of IPF patients treated with IFN- $\gamma$ failed to demonstrate a change in clinical parameters for the progression of disease (15). However, in subgroup analysis of IPF patients with pulmonary function findings of forced vital capacity greater than or equal to $55 \%$ and diffusing capacity for carbon monoxide greater than or equal to $35 \%$ of predicted, there was evidence for a significant survival advantage in patients treated with IFN- $\gamma$ (15). Moreover, a recent multicenter trial of IPF patients treated with IFN- $\gamma$ demonstrated changes in expression of biomarkers of fibrosis, angiogenesis, proliferation, immunomodulation, and antimicrobial activity, which supports the notion that IFN- $\gamma$ may affect IPF through multiple pathways (16). In contrast to previous reports suggesting that IPF patients are deficient in IFN- $\gamma$ (17), the multicenter biomarker study of IPF patients treated with IFN- $\gamma$ demonstrated that at baseline the majority of IPF patients had measurable levels of IFN- $\gamma$ mRNA in their lungs (16). The unexpected finding of this study was that IPF patients treated with IFN- $\gamma$ demonstrated induction of only one of the three CXCR3 ligands: CXCL11, not CXCL9 or CXCL10. This suggests that the potential defect in
IPF may not be directly related to IFN- $\gamma$, but rather related to the failure to fully mount an appropriate CXCR3/CXCR3 ligand response to injury.

\section{Acknowledgments}

R.M. Strieter and M.P. Keane's work on pulmonary fibrosis is supported by NIH grant P50HL67665.

Address correspondence to: Robert M. Strieter, Departments of Medicine, Pathology, and Pediatrics, Division of Pulmonary and Critical Care Medicine, David Geffen School of Medicine at UCLA, 900 Veteran Avenue, 14-154 Warren Hall, Box 711922, Los Angeles, California 90095-1786, USA. Phone: (310) 794-1999; Fax: (310) 794-1998; E-mail: rstrieter@mednet.ucla.edu.

1. Coultas, D.B., Zumwalt, R.E., Black, W.C., and Sobonya, R.E. 1994. The epidemiology of interstitial lung diseases. Am. J. Respir. Crit. Care Med. 150:967-972.

2. Perez, A., Rogers, R.M., and Dauber, J.H. 2003. The prognosis of idiopathic pulmonary fibrosis. Am. J. Respir. Cell Mol. Biol. 29:S19-S26.

3. du Bois, R. 1997. Diffuse lung disease: a view for the future. Sarcoidosis Vasc. Diffuse Lung Dis. 14:23-30.

4. Jiang, D., et al. 2004. Regulation of pulmonary fibrosis by chemokine receptor CXCR3. J. Clin. Invest. 114:291-299. doi:10.1172/JCI200416861.

5. Katzenstein, A.L., and Myers, J.L. 1998. Idiopathic pulmonary fibrosis: clinical relevance of pathologic classification. Am. J. Respir. Crit. Care Med. 157:1301-1315.

6. American Thoracic Society; European Respiratory Society. 2002. American Thoracic Society/European Respiratory Society international multidisciplinary consensus classification of the idiopathic interstitial pneumonias. This joint statement of the American Thoracic Society (ATS), and the European Respiratory Society (ERS) was adopted by the ATS board of directors, June 2001 and by the ERS Executive Committee, June 2001 [review]. Am. J. Respir. Crit. Care Med. 165:277-304.

7. American Thoracic Society. 2000. Idiopathic pulmonary fibrosis: diagnosis and treatment. International consensus statement. American Thoracic Society (ATS), and the European Respiratory Society (ERS). Am. J. Respir. Crit. Care Med. 161:646-664

8. Zuo, F., et al. 2002. Gene expression analysis reveals matrilysin as a key regulator of pulmonary fibrosis in mice and humans. Proc. Natl. Acad. Sci. U. S. A. 99:6292-6297.

9. Hunninghake, G.W., et al. 2003. Radiologic findings are strongly associated with a pathologic diagnosis of usual interstitial pneumonia. Chest. 124:1215-1223.

10. Farber, J.M. 1997. Mig and IP-10: CXC chemokines that target lymphocytes. J. Lenkoc. Biol. 61:246-257.

11. Rabin, R.L., et al. 1999. Chemokine receptor responses on $\mathrm{T}$ cells are achieved through regulation of both receptor expression and signaling. J. Immunol. 162:3840-3850.

12. Qin, S., et al. 1998. The chemokine receptors CXCR3 and CCR5 mark subsets of T cells associated with certain inflammatory reactions. J. Clin. 
Invest. 101:746-754.

13. Loetscher, M., Loetscher, P., Brass, N., Meese, E., and Moser, B. 1998. Lymphocyte-specific chemokine receptor CXCR3: regulation, chemokine binding and gene localization. Eur. J. Immunol. 28:3696-3705.

14. Schroder, K., Hertzog, P.J., Ravasi, T., and Hume, D.A. 2004. Interferon-gamma: an overview of sig- nals, mechanisms and functions. J. Leukoc. Biol. 75:163-189.

15. Raghu, G., et al. 2004. A placebo-controlled trial of interferon gamma- $1 \mathrm{~b}$ in patients with idiopathic pulmonary fibrosis. N. Engl. J. Med. 350:125-133. 16. Strieter, R.M., Starko, K.M., Enelow, R.I., Noth, I., and Valentine, V.G. 2004. Effects of interferon gamma-1b on biomarker expression in idiopathic pulmonary fibrosis patients. Am. J. Respir. Crit. Care Med. doi:10.1164/rccm.200312-1670OC

17. Ziesche, R., Hofbauer, E., Wittmann, K., Petkov, V., and Block, L.H. 1999. A preliminary study of long-term treatment with interferon gamma$1 \mathrm{~b}$ and low-dose prednisolone in patients with idiopathic pulmonary fibrosis. N. Engl. J. Med. 341:1264-1269.

\title{
Adaptive cellular immunity in aortic aneurysms: cause, consequence, or context?
}

\author{
John A. Curci'1,2 and Robert W. Thompson ${ }^{1,2,3}$ \\ ${ }^{1}$ Department of Surgery (Section of Vascular Surgery), ${ }^{2}$ Department of Radiology, and ${ }^{3}$ Department of Cell Biology and Physiology, \\ Washington University School of Medicine, St. Louis, Missouri, USA.
}

\begin{abstract}
Abdominal aortic aneurysms are common and life threatening. Although $\mathrm{CD}^{+} \mathrm{T}$ cells are abundant in aneurysm tissue, their role in disease progression remains unclear. A new study (see the related article beginning on page 300) shows that mouse aortic allografts placed in animals lacking IFN- $\gamma$ receptors develop a Th 2 inflammatory response with aortic aneurysms, whereas Th1 responses promote intimal hyperplasia. It is expected that these surprising findings will stimulate further efforts to clarify whether adaptive cellular immunity in aneurysm disease is detrimental or potentially beneficial.
\end{abstract}

The propensity of abdominal aortic aneurysms (AAAs) to rupture is their most important clinical feature, a consequence of increased hemodynamic stresses placed on the dilated wall, diminished tensile strength within the outer media and adventitia, and dynamic factors influencing the balance between matrix metabolism and repair. Surgical repair of AAAs greater than $5.5 \mathrm{~cm}$ in diameter is effective treatment, but repair of smaller aneurysms offers no survival advantage. Effective nonsurgical treatments to prevent aneurysm expansion would therefore be an enticing prospect for patients with small AAAs (1).

\section{Pathophysiology of AAAs}

Figure 1 presents a summary of pathophysiological events currently thought to contribute to aneurysmal degeneration, based on studies of human end-stage AAA tissues and several different experimental animal

Nonstandard abbreviations used: abdominal aortic aneurysm (AAA); IFN- $\gamma$ receptor deficient (GRKO); smooth muscle cell (SMC); tissue inhibitor of metalloproteinase (TIMP).

Conflict of interest: The authors have declared that no conflict of interest exists.

Citation for this article:

J. Clin. Invest. 114:168-171 (2004)

doi:10.1172/JCI200422309. models. Although the specific etiology is still unclear, aneurysms are probably initiated by aortic wall injury coupled with a series of epidemiological risk factors. Recruitment of leukocytes into the aortic media appears to be an early and pivotal event, likely prodation peptides (3). Mononuclear phagocyte infiltration is associated with production of proinflammatory cytokines (4), prostaglandin derivatives (5), and reactive oxygen species (6) as part of an innate inflammatory response. These macrophages are the principle source of MMPs (7), which can also be secreted by neutrophils, lymphocytes, and resident mesenchymal cells. Gelatinase B (MMP-9) has been extensively studied in human AAAs (8), but many other MMPs and endogenous tissue inhibitors of metalloproteinases (known as TIMPs) have also been described. Animal models of aortic aneurysm confirm that MMPs produced by chronic inflammatory cells are mediators of elastin and collagen degradation (9-11); moreover, the suppression of experimental aneurysms by MMP inhibitors has led to a promising therapeutic strategy (12). Other enzymes expressed in atherosclerosis and AAAs, particularly plasminogen activators and cathepsins, may also contribute to matrix proteolysis. moted by chemokines (2) and elastin degra-
Degradation of elastin and interstitial collagen initiates aortic dilatation and tortuosity, with changes in aortic wall geometry increasing cyclic strain and wall tension over a period of years. At later stages of disease, disorganized interstitial collagen is deposited within the media and adventitia, and collagen degradation becomes more prominent, further weakening the aortic wall. Although medial smooth muscle cells (SMCs) might otherwise promote structural repair in the damaged aorta, apoptosis and cellular senescence cause depletion of this cell population $(13,14)$.

\section{Adaptive immunity in aortic aneurysms}

In addition to macrophages, human AAAs demonstrate large numbers of $\mathrm{T}$ cells, B lymphocytes, plasma cells, and DCs within the outer media and adventitia $(15,16)$. AAA tissues also contain large amounts of immunoglobulin protein, and IgG extracted from human AAAs exhibits immunoreactivity with aortic wall matrix proteins (17). This suggests that a humoral (auto)immune response is a frequent occurrence in AAAs. Recent work has led to identification of several putative antigens that may be novel extracellular matrix proteins associated with large arteries (18).

The specificity of the immune response in AAAs is still unclear, as B lymphocytes derived from AAAs exhibit an unrestricted repertoire of immunoglobulin heavy chain genes (19), and $T$ cell receptor diversity reflects a polyclonal response (20). In a recent and comprehensive analysis, Ocana et al. (21) demonstrated that aneurysm-infiltrating lymphocytes consist of activated memory cells express- 